\title{
Isolation and Characterization of Yeasts Able to Assimilate Sugarcane Bagasse Hemicellulosic Hydrolysate and Produce Xylitol Associated with Veturius transversus (Passalidae, Coleoptera, and Insecta)
}

\author{
Italo Thiago Silveira Rocha Matos, Enedina Nogueira Assunção, Edson Junior do Carmo, \\ Verena Makaren Soares, and Spartaco Astolfi-Filho
}

Departamento de Genética, Universidade Federal do Amazonas, Av. Gal. Rodrigo Octávio, 3000 Manaus, AM, Brazil

Correspondence should be addressed to Italo Thiago Silveira Rocha Matos; italo_matos@ufam.edu.br

Received 6 April 2017; Accepted 10 May 2017; Published 6 June 2017

Academic Editor: Giuseppe Comi

Copyright ( 2017 Italo Thiago Silveira Rocha Matos et al. This is an open access article distributed under the Creative Commons Attribution License, which permits unrestricted use, distribution, and reproduction in any medium, provided the original work is properly cited.

\begin{abstract}
Yeasts are an important component of insect gut microbial content, playing roles such as degradation of polymers and toxic compounds, biological control, and hormone, vitamin, and digestive enzyme production. The xylophagous beetle gut is a hyperdiverse habitat and a potential source of new species with industrial abilities such as enzyme production, pentose fermentation, and biodetoxification. In this work, samples of Veturius transversus (Passalidae, Coleoptera, and Insecta) were collected from the Central Amazon Rainforest. Their guts were dissected and a total of 20 microbial colonies were isolated using sugarcane bagasse hemicellulosic hydrolysate. They were identified as having 10 distinct biochemical profiles, and genetic analysis allowed identification as three clades in the genera Candida, Williopsis, and Geotrichum. All colonies were able to assimilate D-xylose and 18 were able to produce xylitol, especially a strain of Geotrichum, with a maximum yield of $0.502 \mathrm{~g} \cdot \mathrm{g}^{-1}$. These results agree with a previous prediction that the microbial community associated with xylophagous insects is a promising source of species of biotechnological interest.
\end{abstract}

\section{Introduction}

Yeasts are microorganisms of the Fungi kingdom, distributed in the phyla Ascomycota, Basidiomycota, and Deuteromycota [1]. On the other hand, beetles are the most abundant order of insects (Coleoptera, Insecta, Arthropoda, and Metazoa), with more than 400,000 species currently described [2].

The association of yeasts with beetles was decisive in the evolutionary success of these insects, the microbiota being indispensable to them, playing fundamental roles such as in the synthesis of amino acids, lipids, pheromones, and digestive enzymes and in biodetoxification [3,4]. According to Suh et al. [5], the microbial content of a xylophagous beetle's gut is a hyperdiverse source of undescribed species.

Xylitol is a promising polyol of five carbons, with medical applications in middle ear otitis [6] and obesity prevention [7].
It is obtained from D-xylose reduction, performed by microbial fermentation or a chemical process [8]. As this latter releases a high number of by-products, demanding several steps for purification, sampling efforts aiming to isolate microbes with ability to produce xylitol keep being necessary.

Recent yeast and yeast-like fungi sampling efforts have increased the number of known species and strains able to produce vitamins, enzymes, and other products from fermentation of sugars such as ethanol and xylitol [9]. In this way, isolation and characterization of wild-type yeasts and yeast-like fungi remains an important approach.

The aim of this work was to isolate and characterize yeasts associated with the xylophagous beetle Veturius transversus (Passalidae, Coleoptera, and Insecta) able to assimilate sugarcane bagasse hemicellulosic hydrolysate $(\mathrm{SBHH})$ as sole carbon source and produce xylitol by $\mathrm{D}$-xylose fermentation. 


\section{Material and Methods}

Under authorization (protocol number 34652-1) of the Instituto Chico Mendes de Conservação da Biodiversidade (Brazilian authority for biodiversity access), 15 beetles were collected from the Central Amazon Rainforest $\left(3^{\circ} 06^{\prime} 05.20^{\prime \prime}\right.$ $\left.\mathrm{S}, 59^{\circ} 58^{\prime} 23.14^{\prime \prime} \mathrm{W}\right)$. They were identified as V. transversus, a highly representative passalid beetle in this region. Three samples were deposited in the Entomological collection Paulo Burnheim (UFAM, Brazil).

The beetles were washed in $70 \%$ ethanol for $1 \mathrm{~min}$, the elytra were removed, and the gut was dissected. Fragments of intestine of about $1 \mathrm{~cm}$ were incubated for $48 \mathrm{~h}(120 \mathrm{rpm}$, $28^{\circ} \mathrm{C}$ ) in tubes with $10 \mathrm{~mL}$ of $\mathrm{SBHH}$, prepared as previously described $[10,11]$. After this time, $100 \mu \mathrm{L}$ of this suspension was spread on $\mathrm{SBHH}$ added to agar. Yeasts and yeast-like colonies were isolated in Petri dishes containing Sabouraud agar (yeast extract, $10 \mathrm{~g} / \mathrm{L}$; glucose, $40 \mathrm{~g} / \mathrm{L}$; agar, $20 \mathrm{~g} / \mathrm{L}$ ).

To evaluate their ability to ferment D-xylose, a loopful of each isolate was cultured in tubes containing $10 \mathrm{~mL}$ of YNBX medium (yeast nitrogen base without amino acids, $6.7 \mathrm{~g} / \mathrm{L} ; \mathrm{D}$-xylose, $40 \mathrm{~g} / \mathrm{L}$ ). After 7 days of incubation at $28^{\circ} \mathrm{C}$ and $120 \mathrm{rpm}$, the medium content was centrifuged and the supernatant was analysed by an HPLC system using a Rezex RPM monosaccharide column $(300 \times 7.8 \mathrm{~mm}$, $\mathrm{Pb}^{2+} 8 \%$, Phenomenex). The $\mathrm{D}$-xylose consumption rate (\%) was calculated according to the final and initial D-xylose concentrations. Xylitol yield $\left(\mathrm{g} \cdot \mathrm{g}^{-1}\right)$ was calculated by the ratio xylitol produced:D-xylose consumed.

For taxonomic identification, biochemical characterization was performed using kit ID32C (BioMerieux ${ }^{\circledR}$ ), according to the manufacturer's instructions. The results were plotted in the online application ApiWeb ${ }^{\circledR}$ (https://apiweb.biomerieux.com) for physiological similarity identification.

Furthermore, the isolates were evaluated by genomic internal transcribed spacer (ITS) and ribosomal gene nucleotide sequences. The DNA was extracted according to Harju et al. [12] and amplified by PCR using primers ITS1 (5' TCC GTA GGT GAA CCT GCC $3^{\prime}$ ) and ITS4 (5' TCC TCC GCT TAT TGA TAT GC $\left.3^{\prime}\right)$. The PCR products were used to perform a sequencing reaction using a BigDye ${ }^{\circledR}$ kit (Applied Biosystems), and nucleotide sequences were obtained in an Applied Biosystems 3130 Genetic Analyzer ${ }^{\circledR}$ automatic sequencer.

The obtained sequences were compared to the NCBI database (https://www.ncbi.nlm.nih.gov/) using BLAST (Basic Local Alignment Search Tool) and deposited in GenBank. For phylogenetic relationship analysis, nucleotide sequences were aligned using Clustal-W and analysed by neighbour-joining (bootstrap, 2000 replicas), provided by MEGA 6.0 [13]. Nucleotide sequences from the genomic ITS region of Meyerozyma guilliermondii (GenBank JN974905), Trichosporon mycotoxinivorans (GenBank JX891097), and Scheffersomyces stipitis (GenBank GU256745) were included in the phylogenetic tree as reference groups, this last being the external group.

\section{Results}

A total of 20 colonies were isolated and evaluated, correspondent to 10 species of four genera, Candida (12 isolates),
Cryptococcus (five isolates), Debaryomyces (one isolate), and Geotrichum (two isolates); their biochemical profiles are described in Table 1.

Nucleotide sequence BLAST results identified three different groups, one close to Candida tropicalis, another composed of members of the species Williopsis saturnus, and the third composed of the genus Geotrichum sp. The fragment length was about $550 \mathrm{bp}$ for $C$. tropicalis and $W$. saturnus, containing $18 \mathrm{~S}$ rDNA (partial), ITS1 (complete), 5.8S rDNA (complete), ITS2 (complete), and 28S rDNA (partial). For Geotrichum sp., fragment length was on average $250 \mathrm{bp}$, containing ITS1 (partial), 5.8S rDNA (complete), and ITS2 (partial).

For isolates 07,16 , and 18 , identity greater than or equal to $99 \%$ allows us to conclude that these are members of the species $W$. saturnus. Isolates $03,08,09,10,11$, and 20 presented identity varying from $97 \%$ to $99 \%$ with C. tropicalis, making it admissible that they are closely related to this species, here named clade C. tropicalis. The other isolates presented identity varying from $92 \%$ to $96 \%$ with Geotrichum sp. or Galactomyces spp.

Considering the short length of the fragment, which allows classification only at genus level and the synonymy between Geotrichum and Galactomyces, they were classified as clade Geotrichum sp. The complete BLAST results and GenBank accession number of sequences are presented in Table 2 .

Neighbour-joining phylogenetic analysis endorsed the conclusion about the three groups that clade $W$. saturnus is close to Meyerozyma guilliermondii and Scheffersomyces stipitis, whereas C. tropicalis and Geotrichum sp. are closely related to Trichosporon mycotoxinivorans. The phylogenetic tree is presented in Figure 1.

Fermentation tests indicated that none of the isolates produces ethanol using xylose as carbon source. This result was expected because xylose fermentation to ethanol is an uncommon feature, being presented by less than $1 \%$ of known yeast species [14]. However, most of them were able to produce xylitol, only isolates 12 and 13 (Geotrichum sp.) being unable to do this. The highest yield was observed in isolate 01 (Geotrichum sp.), reaching $0.502{\mathrm{~g} \cdot \mathrm{g}^{-1}}^{-1}$ and consuming $92.6 \%$ of the D-xylose. The complete results are presented in Table 3.

\section{Discussion}

All isolates were able to assimilate D-xylose, a common feature in yeasts able to metabolize $\mathrm{SBHH}$ because this is the most abundant monosaccharide in hemicellulose [15]. Candida is the most representative, but that occurs because there are a great number of asexual phase (anamorph) species classified in this genus, which is a polyphyletic group $[5,16]$.

The other genera, Cryptococcus and Debaryomyces, have remarkable biotechnological potential in incorporation of lipids in their biomass, being reported as oleaginous yeasts $[17,18]$. Suh and Blackwell [19] describe the genus Geotrichum as dimorphic fungi, being anamorphs of the genera Dipodascus and Galactomyces and growing being yeast-like according to environmental conditions. 


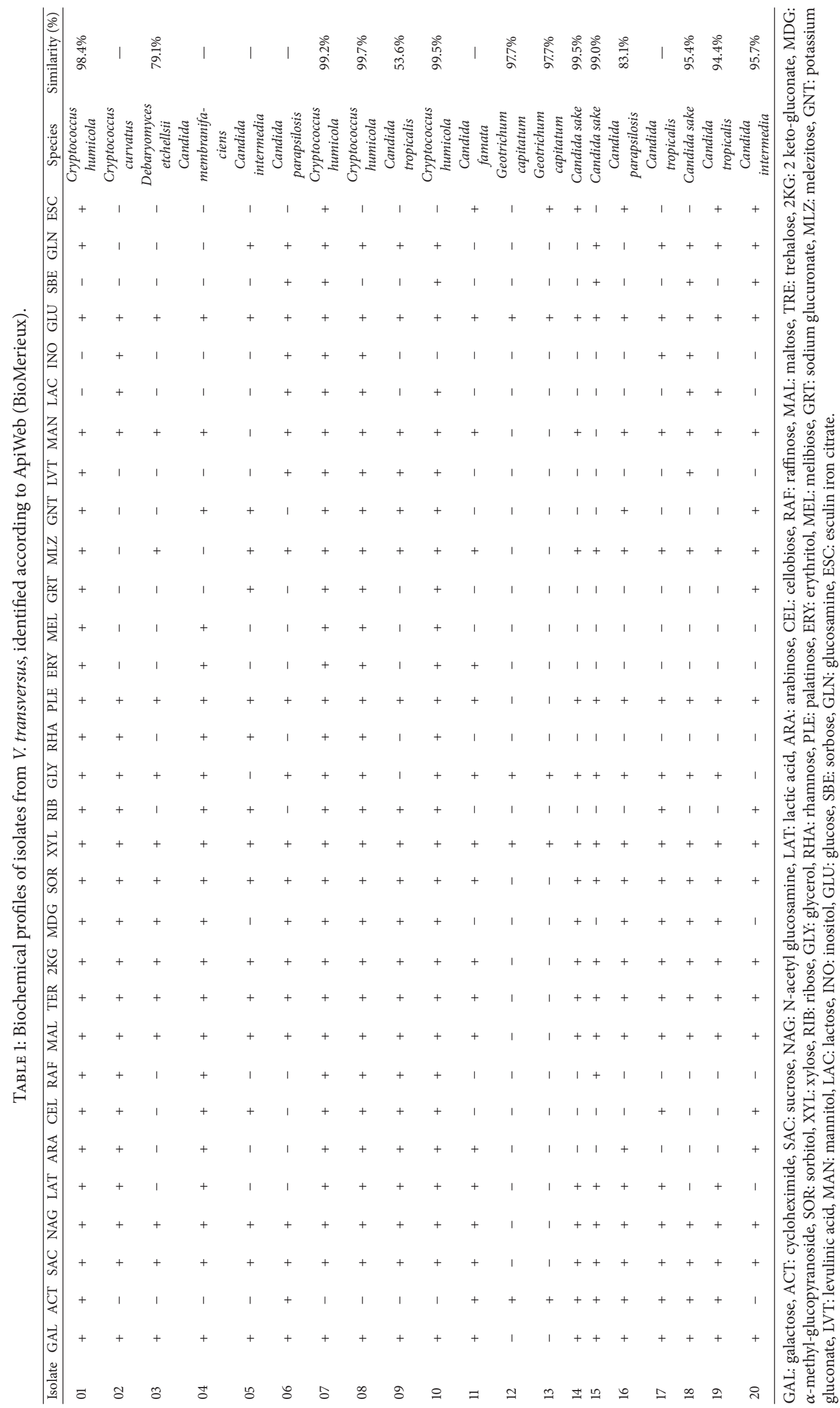


TABLE 2: Identification of isolates according to BLAST result and nucleotide sequence GenBank accession number.

\begin{tabular}{|c|c|c|c|c|c|}
\hline Isolate & Species & Max identity (\%) & Query coverage (\%) & $e$-value & GenBank accession number \\
\hline 01 & Geotrichum sp. & 93 & 92 & $1 e-119$ & KP276644 \\
\hline 02 & Geotrichum sp. & 95 & 93 & $3 e-131$ & KP276636 \\
\hline 03 & Candida tropicalis & 99 & 98 & 0.0 & KP276645 \\
\hline 04 & Geotrichum sp. & 94 & 100 & $1 e-121$ & KP276637 \\
\hline 05 & Galactomyces candidum & 96 & 97 & $1 e-131$ & KP276638 \\
\hline 06 & Geotrichum sp. & 96 & 99 & $1 e-126$ & KP276639 \\
\hline 07 & Williopsis saturnus & 99 & 100 & 0.0 & KP257575 \\
\hline 08 & Candida tropicalis & 98 & 99 & 0.0 & KP276646 \\
\hline 09 & Candida tropicalis & 99 & 99 & 0.0 & KP276647 \\
\hline 10 & Candida tropicalis & 98 & 99 & 0.0 & KP276648 \\
\hline 11 & Candida tropicalis & 98 & 99 & 0.0 & KP276649 \\
\hline 12 & Galactomyces candidum & 96 & 98 & $1 e-136$ & KP276640 \\
\hline 13 & Galactomyces geotrichum & 96 & 98 & $1 e-136$ & KP276641 \\
\hline 14 & Geotrichum sp. & 95 & 100 & $1 e-125$ & KP276642 \\
\hline 15 & Geotrichum sp. & 94 & 100 & $3 e-102$ & KP288488 \\
\hline 16 & Williopsis saturnus & 99 & 100 & 0.0 & KP257574 \\
\hline 17 & Geotrichum sp. & 93 & 99 & $7 e-129$ & KP288487 \\
\hline 18 & Williopsis saturnus & 99 & 100 & 0.0 & KP257573 \\
\hline 19 & Geotrichum sp. & 94 & 99 & $5 e-134$ & KP276643 \\
\hline 20 & Candida tropicalis & 97 & 99 & 0.0 & KP276650 \\
\hline
\end{tabular}

TABLE 3: D-xylose consumption rate (\%) and xylitol yield of each isolate.

\begin{tabular}{lcc}
\hline Isolate & D-xylose consumption rate $(\%)$ & Xylitol yield $\left(\mathrm{g} \cdot \mathrm{g}^{-1}\right)$ \\
\hline 01 & 92.6 & 0.502 \\
02 & 29.9 & 0.210 \\
03 & 33.4 & 0.214 \\
04 & 36.2 & 0.255 \\
05 & 30.7 & 0.186 \\
06 & 35.6 & 0.224 \\
07 & 31.0 & 0.169 \\
08 & 33.2 & 0.180 \\
09 & 34.0 & 0.170 \\
10 & 30.7 & 0.100 \\
11 & 100.0 & 0.339 \\
12 & 27.5 & 0.0 \\
13 & 18.7 & 0.0 \\
14 & 100.0 & 0.315 \\
15 & 100.0 & 0.326 \\
16 & 100.0 & 0.304 \\
17 & 41.4 & 0.304 \\
18 & 100.0 & 0.341 \\
19 & 100.0 & 0.370 \\
20 & 100.0 & 0.347 \\
\hline
\end{tabular}

All biochemical profile results presented similarity with species able to perform pentose fermentation and/or another process with biotechnological potential. However, according to Barnett [20], biochemical profiles may be used as complementary information but cannot be conclusive for taxonomic identification because they can present high variation, with it being recommended to evaluate genomics data. According to Hou-Rui et al. [21], up to $1 \%$ of nucleotide substitution in a ribosomal domain is permitted for strains of a single biological species, rDNA sequence analysis being a simple and reliable tool for taxonomic identification.

The phylogenetic analysis endorses that predicted by Barnett [20], noticeable because clade $W$. saturnus is composed of isolates with three different biochemical profiles, whereas clade C. tropicalis is composed of five different biochemical profiles (one of those C. tropicalis) and clade Geotrichum has eight different biochemical profiles. Furthermore, there were some isolates with the same biochemical profile distributed in all clades, strengthening that hypothesis.

The maximum theoretical yield for xylitol production from D-xylose fermentation is $1.0 \mathrm{~g} \cdot \mathrm{g}^{-1}$. Despite this, as microbes produce xylitol as a compatible solute, it is excreted in osmotic stress conditions and then consumed as the medium becomes less harsh [22]; common yields range from $40 \%$ to $70 \%$ [23]. The highest yield value for microbial fermentation is reported by Granström et al. [24] for Candida sp., at $0.85 \mathrm{~g} \cdot \mathrm{g}^{-1}$.

With Geotrichum sp. (isolate 01) being a wild-type strain with the capability to produce xylitol like some industrial strains, it can be considered a promising xylitol-producing yeast. This is the first work to report xylitol production by wild-type yeast strains associated with beetles from the Central Amazon Rainforest. 


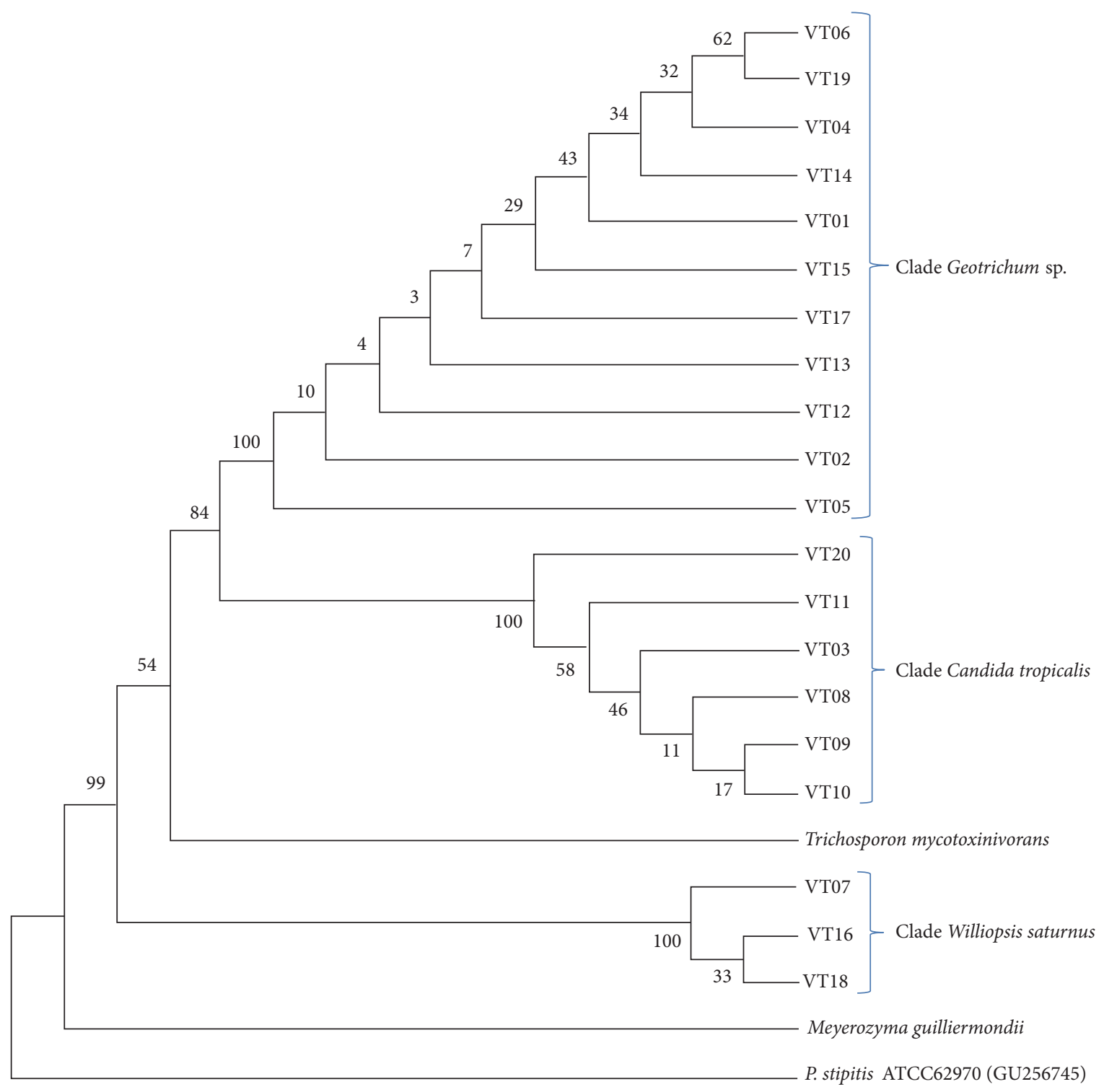

FIGURE 1: Neighbour-joining phylogenetic tree, endorsing the distribution of the isolates into three groups.

\section{Conclusion}

The yeast community associated with $V$. transversus gut is rich in D-xylose-assimilating and xylitol-producing species, some of which present potential close to industrial strains. Geotrichum is a highly representative group in this community.

Geotrichum sp. (isolate 01) presents high xylitol yield, reaching about $50 \%$ of the maximum theoretical yield, and is a promising xylitol-producing strain.

Subsequent efforts must be concentrated on developing bioprocesses using these isolates.

\section{Conflicts of Interest}

The authors declare that there are no conflicts of interest.

\section{Acknowledgments}

Special thanks are due to Coordenação de Aperfeiçoamento de Pessoal de Nível Superior (CAPES), Conselho Nacional de Desenvolvimento Científico e Tecnológico (CNPq), and Fundação de Amparo a Pesquisa do Estado do Amazonas (FAPEAM). The authors are also grateful to Dra. Nair Otaviano Aguiar for beetle identification.

\section{References}

[1] C. J. ALexopoulos, C. W. Mims, and M. Blackwell, Introductory Mycology, John Wiley \& Sons, New York, NY, USA, 4th edition, 1996.

[2] A. S. Lelej and S. Y. Storozhenko, "Insect taxonomic diversity in the Russian Far East," Entomological Review, vol. 90, no. 3, pp. 372-386, 2010. 
[3] T. S. Davis, "The Ecology of Yeasts in the Bark Beetle Holobiont: A Century of Research Revisited," Microbial Ecology, vol. 69, no. 4, pp. 723-732, 2015.

[4] S.-O. Suh, C. J. Marshall, J. V. McHugh, and M. Blackwell, "Wood ingestion by passalid beetles in the presence of xylosefermenting gut yeasts," Molecular Ecology, vol. 12, no. 11, pp. 3137-3145, 2003.

[5] S.-O. Suh, N. H. Nguyen, and M. Blackwell, "Nine new Candida species near C. membranifaciens isolated from insects," Mycological Research, vol. 109, no. 9, pp. 1045-1056, 2005.

[6] A. Azarpazhooh, H. Limeback, H. P. Lawrence, and P. S. Shah, "Xylitol for preventing acute otitis media in children up to 12 years of age," Cochrane database of systematic reviews, no. 11, Article ID CD007095, 2011.

[7] J. C. Parajó, H. Domínguez, and J. M. Domínguez, "Biotechnological production of xylitol. Part 1: Interest of xylitol and fundamentals of its biosynthesis," Bioresource Technology, vol. 65, no. 3, pp. 191-201, 1998.

[8] Z. D. V. L. Mayerhoff, I. C. Roberto, and S. S. Silva, "Xylitol production from rice straw hemicellulose hydrolysate using different yeast strains," Biotechnology Letters, vol. 19, no. 5, pp. 407-409, 1997.

[9] H. Urbina, J. Schuster, and M. Blackwell, "The gut of Guatemalan passalid beetles: A habitat colonized bycellobioseand xylose-fermenting yeasts," Fungal Ecology, vol. 6, no. 5, pp. 339-355, 2013.

[10] I. T. S. R. Matos, L. A. Cassa-Barbosa, R. S. Medeiros Galvão, C. G. Nunes-Silva, and S. Astolfi Filho, "Isolation, taxonomic identification and investigation of the biotechnological potential of wild-type Meyerozyma guilliermondii associated with Amazonian termites able to ferment D-xylose," Bioscience Journal, vol. 30, no. 1, pp. 260-266, 2014.

[11] I. T. S. R. Matos, L. A. Cassa-Barbosa, P. Q. Costa Neto, and S. Astolfi Filho, "Cultivation of Trichosporon mycotoxinivorans in sugarcane bagasse hemicellulosic hydrolyzate," Electronic Journal of Biotechnology, vol. 15, no. 1, 2012.

[12] S. Harju, H. Fedosyuk, and K. R. Peterson, "Rapid isolation of yeast genomic DNA: Bust n' Grab," BMC Biotechnology, vol. 4, article no. 8, 2004.

[13] K. Tamura, G. Stecher, D. Peterson, A. Filipski, and S. Kumar, "MEGA6: Molecular Evolutionary Genetics Analysis version 6.0," Molecular Biology and Evolution, vol. 30, no. 12, pp. 27252729, 2013.

[14] B. Hahn-Hägerdal, H. B. K. Karhumaa, C. Fonseca, I. SpencerMartins, and M. F. Gorwa-Grauslund, "Towards industrial pentose-fermenting yeast strains," Applied Microbiology and Biotechnology, vol. 74, no. 5, pp. 937-953, 2007.

[15] B. C. Saha, "Hemicellulose bioconversion," Journal of Industrial Microbiology and Biotechnology, vol. 30, no. 5, pp. 279-291, 2003.

[16] F. Schauer and R. Hanschke, "Taxonomy and ecology of the genus Candida," Mycoses, vol. 42, no. S1, pp. 12-21, 1999.

[17] J. M. Ageitos, J. A. Vallejo, P. Veiga-Crespo, and T. G. Villa, "Oily yeasts as oleaginous cell factories," Applied Microbiology and Biotechnology, vol. 90, no. 4, pp. 1219-1227, 2011.

[18] E. A. Johnson, "Biotechnology of non-Saccharomyces yeasts The ascomycetes," Applied Microbiology and Biotechnology, vol. 97, no. 2, pp. 503-517, 2013.

[19] S.-O. Suh and M. Blackwell, “Three new asexual arthroconidial yeasts, Geotrichum carabidarum sp. nov., Geotrichum histeridarum sp. nov., and Geotrichum cucujoidarum sp. nov., isolated from the gut of insects," Mycological Research, vol. 110, no. 2, pp. 220-228, 2006.

[20] J. A. Barnett, "A history of research on yeasts 8: Taxonomy," Yeast, vol. 21, no. 14, pp. 1141-1193, 2004.

[21] Z. Hou-Rui, Q. Xiang-Xiang, S. S. Silva et al., "Novel isolates for biological detoxification of lignocellulosic hydrolysate," Applied Biochemistry and Biotechnology, vol. 152, no. 2, pp. 199-212, 2009.

[22] G. M. Walker, Yeast Physiology and Biotechnology, John Wiley \& Sons, New York, NY, USA, 1998.

[23] J. S. Aranda-Barradas, C. Garibay-Orijel, J. A. Badillo-Corona, and E. Salgado-Manjarrez, "A stoichiometric analysis of biological xylitol production," Biochemical Engineering Journal, vol. 50, no. 1-2, pp. 1-9, 2010.

[24] T. B. Granström, K. Izumori, and M. Leisola, "A rare sugar xylitol. Part II: Biotechnological production and future applications of xylitol," Applied Microbiology and Biotechnology, vol. 74, no. 2, pp. 273-276, 2007. 

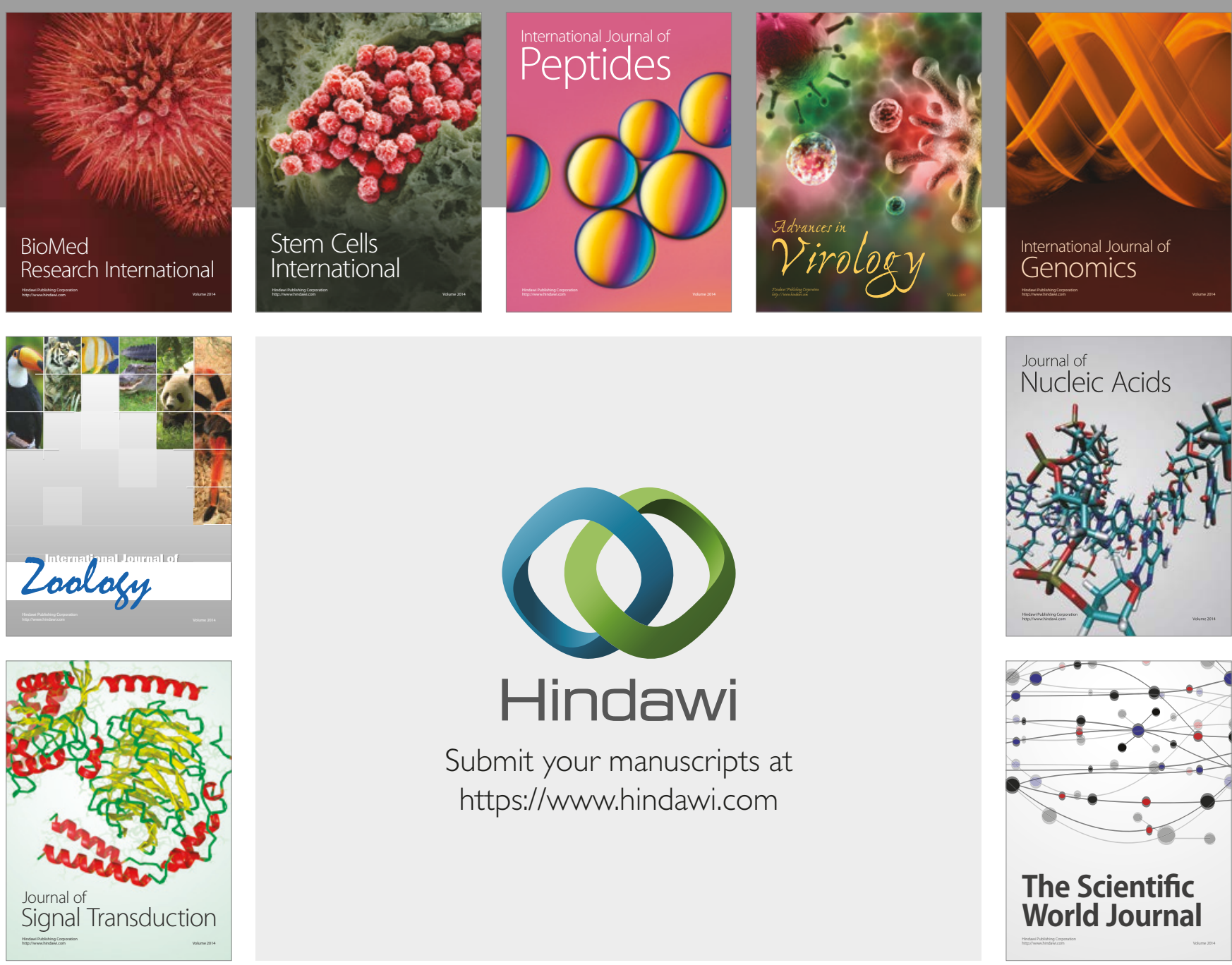

Submit your manuscripts at

https://www.hindawi.com
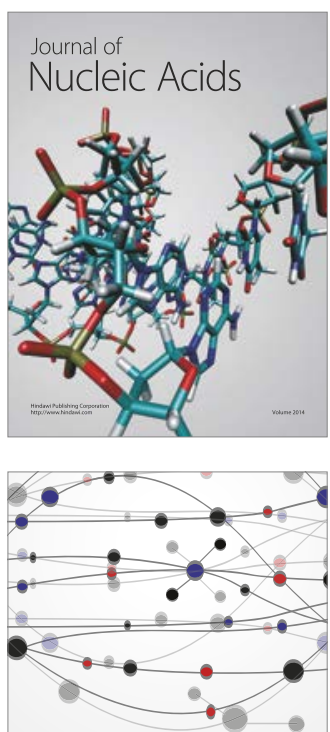

The Scientific World Journal

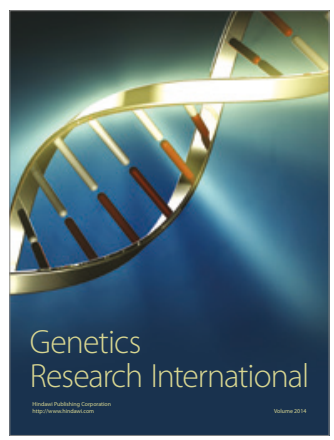

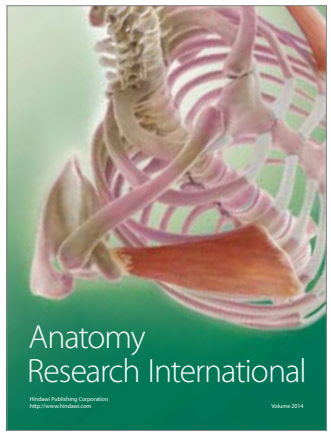

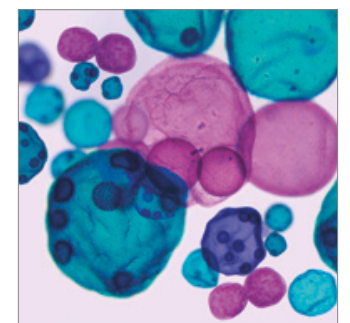

International Journal of Microbiology
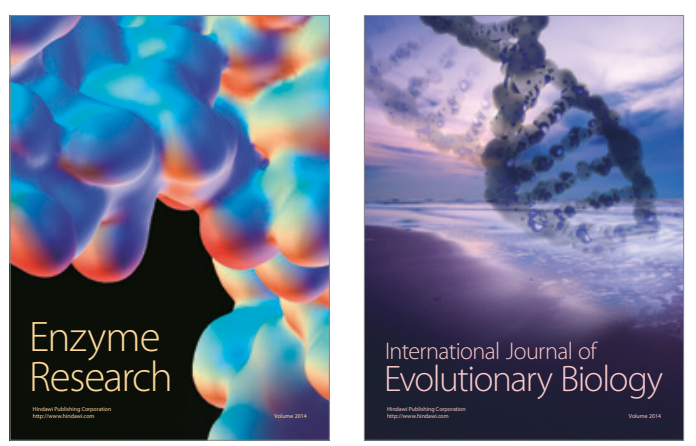
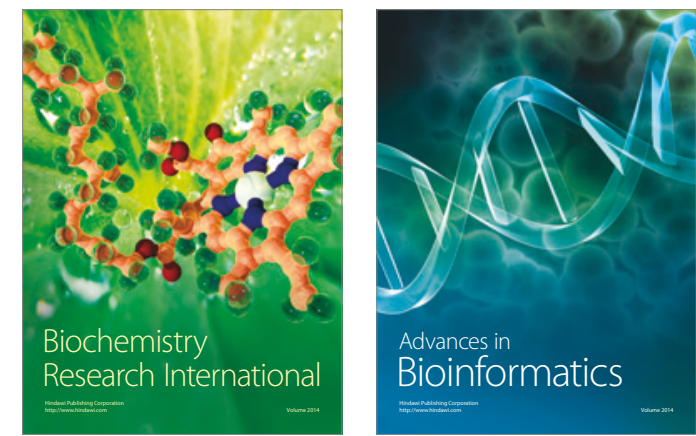

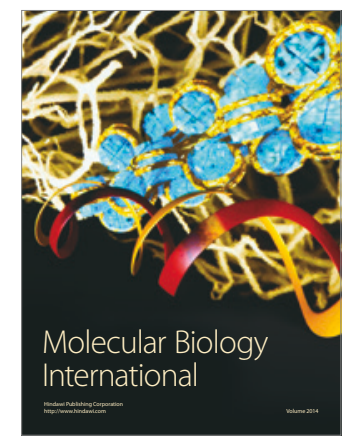

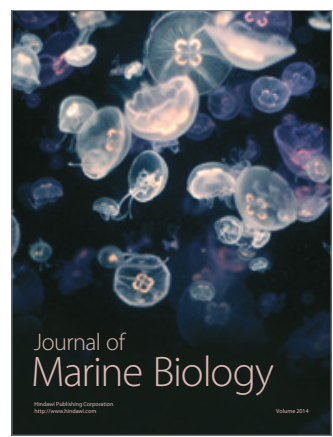

